\title{
¿CÓMO OPERA LA PAREJA PEDAGÓGICA COMO DISPOSITIVO DE FORMACIÓN DOCENTE?
}

\author{
Lucila, Beltramo (Universidad Nacional de Rosario)* \\ lucila_beltramo@hotmail.com
}

Recibido: 24/07/2012 Aceptado: 11/09/2012

\begin{abstract}
Resumen
El presente artículo corresponde a un trabajo de adscripción realizado en la Cátedra de Residencia Docente de la carrera de Ciencias de la Educación, de la Facultad de Humanidades y Artes de la Universidad Nacional de Rosario, en el cual se explicita cómo opera la pareja pedagógica en su vinculación con diferentes dispositivos de formación docente utilizados por la cátedra, como ser: la biografía escolar, los diarios de formación o diarios de clase, la observación y las microclases o prácticas simuladas de enseñanza.

A partir del trabajo con diferentes dispositivos de la formación docente se busca concretar el aprendizaje de la reflexión y de la construcción de conocimiento profesional docente durante la formación inicial.

Las competencias profesionales sólo pueden construirse verdaderamente gracias a una práctica reflexiva y comprometida que se instale desde el principio de nuestros estudios. (Perrenoud, 2006)
\end{abstract}

\section{Palabras clave}

Pareja pedagógica - Dispositivo - Reflexión - Formación - Grupo.

\begin{abstract}
This item is a work assignment performed in the Department of Teaching Residency race Educational Sciences, Faculty of Humanities and Arts at the National University of Rosario, in which explicit how the couple operates pedagogical its relationship with different teacher training devices used by the department, such as: the school
\end{abstract}

* Profesora en Ciencias de la Educación. Becaria de la cátedra de Residencia Docente. Escuela de Ciencias de la Educación. Facultad de Humanidades y Artes. Universidad Nacional de Rosario. 
biography, diaries or journals class training, observation and simulated microclasses or teaching practices.

From the work with different devices of teacher seeks concrete reflection learning and teacher professional knowledge construction during initial training.

The skills really only be built through reflective practice and committed to be installed from the beginning of our studies. (Perrenoud, 2006)

\section{Key words}

Couple pedagogical - Device - Reflection - Training - Group.

\section{Introducción}

El presente artículo titulado “¿Cómo opera la Pareja Pedagógica como dispositivo de formación docente?" corresponde a un trabajo de adscripción realizado en la Cátedra de Residencia Docente de la carrera de Ciencias de la Educación, de la Facultad de Humanidades y Artes de la Universidad Nacional de Rosario.

La pareja pedagógica conformada por residentes, como dispositivo de formación, consiste en el acompañamiento que realizan entre pares en el período que comienzan a desarrollar una Unidad Didáctica. Este acompañamiento favorece la reflexión sobre las propias prácticas, ayuda a enfrentar diferentes situaciones y emociones, ya sean positivas como negativas. La significatividad que tiene trabajar de esta manera en la residencia docente se ve reflejada a continuación en los siguientes párrafos extraídos de entrevistas realizadas a profesoras egresadas de la cátedra:

(...) la pareja pedagógica te da la posibilidad de trabajar en equipo, con un par, algunas de las tareas de la Residencia. Esto implica que alguien de quien yo me siento cercano y con el cual estoy atravesando una experiencia similar pueda acompañar mi proceso y realizar comentarios y sugerencias. De la misma manera, yo podré ir acompañando el proceso de mi pareja y, a la vez que intento ir haciéndole aportes, voy teniendo en cuenta su experiencia para iluminar de alguna manera la mía. (Residente, 2009)

Me aportó la mirada de quien se estaba formando como yo y que podía opinar con el compromiso de quien está transitando por esa misma experiencia. Es decir, que en muchas ocasiones los aportes de una y otra se volvía también aportes para la propia práctica, lo que yo podía visualizar en la práctica de mi compañera se transformaba en un aspecto a tener en cuenta en mi práctica posterior y viceversa. Aunque en cada desempeño se evidenciaba una, manera propia y particular de llevar a delante la clase, los comentarios de una compañera de trabajo, enriquecía la planificación y abría nuevas posibilidades. (Residente, 2008) 
Es un dispositivo de aprendizaje fundamental, tanto para la formación de los residentes, como para su seguimiento, contención y acompañamiento. Puesto en acto de un modo correcto es un dispositivo que promueve cambios al permitirle al residente hacer conciente su práctica pedagógica, reflejada en la narrativa y palabras de su compañero, lo necesariamente significativas como para reflejarse en ellas.

Al interior de este binomio se establece un vínculo pedagógico diferenciado, único y específico de aquél que se constituye con el formador y el co-formador.

Considero, que los procesos básicos que atraviesan a esta peculiar relación pedagógica son el diálogo, la comunicación y la discusión reflexiva. Procesos que trabajados adecuadamente, previamente a poner en marcha este dispositivo, logra una construcción. (Residente, 2007).

Considerando el alto impacto que tiene la pareja pedagógica, proponemos en el presente artículo incorporar su reflexión articulada con los diferentes dispositivos de formación docente incluidos en el proyecto de cátedra, como ser: la biografía escolar; los diarios de formación o diario de clase; la observación y las microclases: prácticas simuladas de enseñanza.

Entendemos al dispositivo como:

(...) aquellos espacios, mecanismos, engranajes o procesos que facilitan, favorecen o pueden ser utilizados para la concreción de un proyecto o la resolución de problemáticas. El dispositivo ejerce poder, pero también crea condiciones para analizarlo. Y además, persigue el objetivo de promover en otros la disposición, abre el juego de potencialidades creativas, tiene la intencionalidad de provocar cambios (Sanjurjo, Caporossi, España, Hernández, Alfonso \& Foresi, 2009: 32-33).

El trabajo con los dispositivos se realiza dentro de los espacios de las comisiones, las cuales se conforman con residentes que llevan a cabo su práctica en una misma institución, coordinadas por un profesor de la cátedra y la colaboración de adscriptos. Este espacio permite que los residentes comiencen a retrabajar sus prácticas y logren reflexionar desde la acción y sobre la acción. 


\section{Marco teórico}

"Los residentes de la docencia deben estar preparados no solamente en las herramientas teóricas y prácticas, sino también en actitudes de autonomía, compromiso y pasión para enfrentar las situaciones que se presentan".

Liliana Sanjurjo (2009)

Tradicionalmente la Formación Docente ha respondido al modelo positivista: primero se aprendía la teoría y luego la misma se ponía en práctica. Se trataba de "una visión ingenieril de la teoría que supone la posibilidad de abordaje neutral y control absoluto de las prácticas y coloca al docente en el papel de técnico que aplica teorías" (Sanjurjo, 2005, p. 20).

Hoy, en la Cátedra de Residencia Docente de la Carrera de Ciencias de la Educación de la Facultad de Humanidades y Artes de la Universidad Nacional de Rosario, entendemos la formación de grado como el proceso a través del cual se produce una apropiación del conocimiento científico y tecnológico de una disciplina específica, la reelaboración de una cultura del trabajo profesional y el dominio de competencias profesionales específicas. (Sanjurjo, Hernández, Caporossi, España, Foresi, Alfonso \& Hernández, 2009, p. 10)

Desde este enfoque la Cátedra entiende la residencia docente no como un apéndice de la formación teórica previa, en la que se pone en acto lo aprendido con anterioridad, sino como un espacio de construcción reflexiva, en el cual la lectura de la práctica y la interacción paulatina con la misma juegan un rol fundamental.

Trabajar sobre las prácticas de los residentes para transformarlas nos exige formar a un practicante reflexivo que es ante todo formar a un "(...) profesional capaz de dominar su propia evolución, construyendo competencias y saberes nuevos o más precisos a partir de lo que ha adquirido y de la experiencia" (Perrenoud, 2006, p. 23).

Para lograr que los residentes adquieran una práctica reflexiva y con ella puedean analizar sus prácticas, se considera interesante la propuesta de $\mathrm{Pe}$ rrenoud (2006) de realizar un trabajo de grupo. Entendiendo al grupo como un conjunto de personas que llevan a cabo una práctica o que se están formando para ésta en un dispositivo de alternancia reuniéndose alrededor de un profesor. El cual deberá limitarse a fomentar y a proporcionar las herramientas necesarias para un trabajo de autoanálisis, y ayudar a los estudiantes a explicitar y a interpretar sus prácticas. "El interés del análisis de la práctica en grupo es que cada uno pueda contribuir a interrogar al otro, a sugerir pistas y a matizar la interpretaciones" (Perrenoud, 2006, p. 119).

La participación de los residentes en un grupo de análisis puede funcionar como iniciación a una práctica reflexiva personal, como así también para que 
ellos mismos como futuros docentes las generen en las instituciones en las que trabajen, ya que la propuesta de Perrenoud es que este trabajo en grupo comience en los primeros años del profesorado y continúe hasta el último.

El análisis de la práctica en la Cátedra de Residencia Docente se lleva a cabo dentro de las comisiones en donde se enriquece además, con la utilización de los diferentes dispositivos para la formación docente que intentan concretar el aprendizaje de la reflexión y de la construcción de conocimiento profesional docente durante la formación inicial

Dado que el dispositivo de la pareja pedagógica adquiere significación en el marco de los otros, a continuación nos referiremos a cómo opera en su vinculación con los diferentes dispositivos de la formación docente utilizados en la cátedra.

\section{La biografía escolar}

En la Cátedra de Residencia los alumnos realizan dos trabajos prácticos relacionados entre sí, ambos se refieren a la escritura de su biografía escolar, entendiendo a la misma como "(...) un relato que refiere a los aprendizajes incorporados en el paso por la escolaridad, en todo ese trayecto en el que como sujetos estamos insertos en las instituciones educativas" (Caporossi, 2009, p. 113). Con este trabajo se pretende que los residentes al relatar su biografía puedan acceder a un conocimiento de sí mismo más profundo, identificar sus fortalezas y debilidades, hacer visibles ciertas dimensiones que en una mirada superficial se vuelven oscuras, vincular aspectos ligados a la vida personal y profesional, y establecer relaciones entre la teoría pedagógica adquirida durante la carrera y las propias experiencias escolares.

La biografía escolar es trabajada tanto por cada uno de los grupos en las reuniones de comisión como con la pareja pedagógica con el objetivo de que cada uno reconozca su propia práctica y de esta manera logre comprender futuras prácticas, pensamientos o acciones.

La observación de la pareja pedagógica favorece la confrontación de lo que el residente hace en la clase y lo que explicitó en su biografía escolar, si reproduce o no modelos de docentes y actitudes internalizadas durante su biografía escolar.

Coincidimos con Alliaud (2009) cuando plantea que el recorrido que transitaron los docentes para llegar nuevamente a la escuela ejerció "influencia" en su ser como docentes. Este recorrido comprende los distintos niveles escolares por los que debió transitar, la formación inicial y los primeros desempeños laborales. Recordemos que sus estudios se refieren a cómo la biografía escolar "impactó", "marcó", "dejó huellas", "ejemplos" (para seguir o no), "modelos" y "contramodelos" en los docentes principiantes.

Consideramos que la siguiente cita resalta el impacto que tiene la biografía escolar sobre la construcción del conocimiento profesional docente: 
El primer tiempo es un tiempo de acomodación en el que serán bosquejados distintos modelos de desempeño. Muchos actuarán por imitación, tratando de asemejarse a los personajes vividos como ideales en el transcurso de su paso por las sucesivas instituciones escolares y de formación profesional. Otros actuarán por contraste u oposición, tratando de diferenciarse de aquellos personajes que marcaron negativa o dolorosamente su propia trayectoria. Con voluntad y con suerte con el tiempo iremos construyendo como un collage, nuestro propio estilo (Frigerio y otros, 1992 citado en Alliaud \& Antelo, 2009, p. 52).

La utilización de la pareja pedagógica como dispositivo de formación articulado con la escritura de la biografía escolar promueve una mayor reflexión y análisis sobre la práctica pedagógica, los modelos internalizados acríticamente y permite además un trabajo profundo sobre las propias experiencias.

\section{Diarios de formación o diario de clase}

El diario de clase es un documento personal y autobiográfico del docente, es un registro que incluye opiniones, sentimientos, interpretaciones, reflexiones acerca de las prácticas pedagógicas puestas en acto en el salón de clase. Se lo utiliza como instrumento para la construcción reflexiva del conocimiento profesional y como recurso para el análisis de la teoría o supuestos que sustentan las decisiones docentes, en tanto provee una base comprensiva y rica para el estudio del proceso de enseñanza. La importancia del diario de clase se profundiza cuando las narrativas en él presentes se comparten con otros, cuando su análisis se lleva a cabo de modo colegiado (Sanjurjo, Caporossi, España, Hernández, Alfonso \& Foresi, 2009, p. 132).

En la Cátedra de Residencia Docente se utiliza este dispositivo para que los residentes tomen nota de las observaciones que realizan al profesor coformador que los recibe en su aula para que lleven a cabo sus prácticas. También se utiliza para que lleven un registro diferido de sus clases anotando lo que sucedió en ellas, posibles reflexiones, autocríticas, cuestiones a tener en cuenta para mejorar su práctica, etc.

En las comisiones, se analizan los diarios de clase. En ese trabajo de análisis se confrontan los diarios con las observaciones del profesor de la cátedra y de la pareja pedagógica. Estas acciones crean espacios de reflexión sobre la acción y enriquecen la construcción del conocimiento profesional de la práctica docente. Sostenemos que, "La lectura compartida del diario puede permitir que tutores y pares entablen un diálogo con el autor, que este lo retroalimente y le 
permita profundizar su producción. En tal sentido, esta práctica resulta superadora de las limitaciones propias del dispositivo" (Anijovich, et al., 2009: 116).

La lectura compartida de las observaciones realizadas por la pareja pedagógica, posteriormente a la clase, impacta en el registro diferido de la clase tanto del residente que tiene a cargo la clase como del que observa, sistematizando la reflexión sobre la práctica.

Si bien el diario es un documento personal, y en este sentido puede dar cuenta de aspectos íntimos que su autor tiene derecho a resguardar para sí mismo, es interesante pedir a los autores de diarios de formación que elijan algunos pasajes para leer en voz alta con un tutor o grupos de pares. Este ejercicio puede ser revelador al compartir la lectura con personas que estuvieron presentes en la clase a la que se hace referencia, o que también están transitando el trayecto de formación (Anijovich, et al., 2009, p. 115)

Consideramos que la escritura del diario de clase es muy importante porque permite volver a mirar las prácticas pasadas, a partir de su lectura y relectura con la pareja pedagógica y con el grupo en las comisiones, favoreciendo de esta manera, generar un compromiso con la práctica futura. Esas re-lecturas posibilitan una comprensión del proceso realizado, lo que genera nuevos conocimientos profesionales.

\section{La observación}

Coincidimos con Alfonso (2009) quien entiende a la observación como un instrumento de análisis de las situaciones escolares que nos posibilita indagar, develar, descubrir para comprender la complejidad escolar que queremos conocer. Cabe aclarar que la observación no es neutral, porque el que ve es el sujeto, culturalmente situado y socializado, con determinadas experiencias y conocimientos. Con lo cual podemos decir que la biografía escolar ejerce un fuerte impacto a la hora de realizar una observación.

En la residencia docente la observación es participante, ya que se produce la interacción social entre el observador y los observados en su contexto porque el residente, mientras observa, es integrado al aula como colaborador del docente observado, y es mediante dicha observación que se recogen datos de modo sistemático. Es por eso que el observador entra al campo (sin hipótesis o pre-conceptos cerrados) con interrogantes, objetivos amplios y un sustento teórico construido desde su propio recorrido académico (Sanjurjo, Caporossi et al., 2009).

Acordamos con Aebli (1995) en cuanto a que "(...) no existe una educación de la aptitud de observación en general, pero habría de capacitar al alumno para reconocer los datos esenciales de cualquier hecho" (p. 98), porque la observación implica un proceso de aprendizaje. Es importante que el residente 
en ese proceso aprenda a hacer foco, evitando describir prácticamente todo lo que sucede en el aula, para lograr, como lo hacen los expertos, distinguir lo esencial y más significativo y rechazar lo poco trascendente.

Al respecto Santos Guerra (1998) manifiesta que "No se trata sólo de mirar, sino de buscar (...) Este proceso de conocimiento no exige solamente un registro fiel de lo que sucede, sino una exploración intencionada que descubra la interpretación de lo que acontece" (p. 89).

Jackson (1991 citado en Anijovich, et al., 2009), afirma que se trata de aprender a observar desde la extranjeridad, rompiendo con la somnolencia, es decir, que en tanto observadores debemos revisar, reflexionar y deconstruir las naturalizaciones ya establecidas desde los inicios de nuestro trayecto escolar.

En la cátedra de Residencia Docente se trabaja con los alumnos para que observen y registren en las clases en las que realizan sus residencias: las estrategias de enseñanza, el tipo de conversación que se establece entre el docente y los alumnos, las acciones del docente, las tareas que realizan los alumnos, el uso del pizarrón, la organización y secuenciación de los contenidos que se enseñan, etc.

Este dispositivo es utilizado a lo largo de todo el año. El residente realiza observaciones al coformador y a la pareja pedagógica. Lo cual implica un gran compromiso porque la información que releva la utiliza en los diferentes momentos de la clase, preactivo, interactivo y postactivo.

En síntesis, la articulación de dispositivos como la observación y la pareja pedagógica impactan en la formación de la práctica reflexiva, en tanto permiten volver sobre la práctica no en soledad, sino acompañado.

\section{Microclases: prácticas simuladas de enseñanza}

Otro dispositivo utilizado por la cátedra de Residencia Docente es la Microclase.

Consideramos a la microclase:

Como un dispositivo de formación con características singulares que permite tanto la realización de prácticas, comprendiendo sus fundamentos como la resolución de problemas y el abordaje de imprevistos. Puede contribuir a la reconstrucción de prácticas rutinizadas y naturalizadas de enseñanza y ayudar a los estudiantes a asumir una posición crítica. (Anijovich, et al., 2009, p. 132).

El origen de este dispositivo se remonta al año 1963, donde un grupo de investigadores de la educación como Fortune, MacDonald, Cooper, Allen, Stround y un grupo de maestros desarrollaron en la Universidad de Stanford una estrategia llamada "microenseñanza" para la formación y el perfeccionamiento de los docentes. 
Como lo planteaban sus primeros autores, el dispositivo estaba ligado a una visión positivista de la formación docente que enfatizaba el desarrollo de habilidades propias de una concepción técnica de la enseñanza como por ejemplo, aprender a usar un pizarrón de manera organizada acompañando la exposición, conducir un interrogatorio didáctico, abrir una clase, usar correctamente la voz y el vocabulario, entre otras.

Actualmente se utiliza desde un paradigma de la formación de docentes reflexivos, prestando especial atención a las decisiones que los docentes toman en el proceso de diseño, coordinación y evaluación de sus propias prácticas de la enseñanza.

En este sentido, la microclase permite generar una práctica reflexiva en los espacios de las comisiones, cuando se trabajan los tres momentos de la clase que plantea $\mathrm{Ph}$. W. Jackson. El primero, la enseñanza preactiva, en la que se planifica la clase que se va a desarrollar; el segundo, la enseñanza interactiva, que es el momento de la interacción con los alumnos, y, por último, la enseñanza postactiva, que se centra en un análisis de lo ocurrido en la clase y en la fase de diseño.

Siguiendo este modelo en las comisiones se trabaja de la siguiente manera:

- La enseñanza preactiva. El residente relata los temas que el coformador le asignó, cómo piensa trabajarlos y armar su planificación. El grupo y la pareja pedagógica lo escucha, le pregunta, generando un espacio de reflexión sobre los conceptos aprendidos y los aspectos de la práctica.

- La enseñanza interactiva. En esta instancia el residente desarrolla la clase planificada al interior de la comisión. Tanto los pares como el profesor de la cátedra registran lo que sucede.

- La enseñanza postactiva. Finalmente se produce un intercambio entre pares y docente. Éste es el momento en el que el residente realiza un trabajo de reconstrucción de significados sobre la clase desarrollada, a partir del diálogo en el que intenta comprender la práctica utilizando las creencias, ideas previas construidas en los trayectos escolares y los conocimientos teóricos aprendidos en los años de cursado de la carrera. Esta instancia se replica cuando el residente reflexiona sobre las clases desarrolladas en situaciones concretas de aulas, a partir de los aportes de la pareja pedagógica, del coformador, del formador y de su propia percepción sobre lo ocurrido (Anijovich, et al., 2009).

Cabe aclarar que no sólo aprende el que participa, sino también sus colegas que observan, analizan la clase y comparten las devoluciones y posterior reflexión sobre la misma. Con esta actividad se busca que el residente no se sienta juzgado, sino contenido, ayudado y de esta manera logre reflexionar sobre su propia práctica. Es interesante que todos los residentes pasen por esta instancia una o varias veces dependiendo de las necesidades de cada uno. 
Trabajar con este dispositivo permite tomar como punto de análisis una situación de enseñanza reciente, realizada en la comisión con un número reducido de participantes, con el fin de tener el tiempo suficiente para una reflexión crítica sobre aspectos como "(...) el tratamiento del contenido, la definición de sus alcances, la pertinencia entre el contenido y las actividades propuestas, las cuestiones propias de su 'acción docente` (por ejemplo, las decisiones que toma, el tipo de diálogo que establece con los alumnos, su estilo personal)" (Anijovich, et al., 2009, p. 130).

\section{Reflexión final}

"Camino junto a otros, luego existo", "Nadie se concientiza sólo", Paulo Freire.

La propuesta de utilización de diferentes dispositivos articulados en la residencia docente contribuye a la formación de profesionales reflexivos. Entendemos a la práctica reflexiva como eje estructurante de la formación docente. Coincidimos con Perrenoud (2006) en que:

La formación de una práctica reflexiva no responde, en sí misma, a la cuestión del sentido. Pero permite plantearla con algunos instrumentos y favorece cierta sabiduría, que consiste en renunciar a las evidencias, a los problemas definitivamente resueltos y a los juicios egocéntricos. El practicante reflexivo nada en la complejidad como un pez en el agua, o por lo menos sin resistencia o nostalgia incurable del tiempo en el que todo era blanco y negro.

Formar a docentes comprometidos con su tarea, abiertos a la mirada de los otros, a la escucha, a la lectura, a la crítica, a la reflexión, es contribuir a mejorar la educación, es intentar romper con el paradigma de las certezas, es preguntarse sobre lo dado, afrontar riesgos, lo inesperado y lo incierto.

En la formación inicial, es importante crear lugares para el análisis de la práctica, de mestizaje, de aportaciones y de reflexión sobre la forma cómo pensamos, decidimos, comunicamos y reaccionamos en una clase. Los espacios de las comisiones en la Cátedra de Residencia Docente, como lugar de encuentro semanal, es el lugar propicio para trabajar con los residentes y lograr que ellos aprendan a reflexionar reflexionando.

Los dispositivos propuestos tendrían la función de generar espacios para comenzar a reflexionar. Coincidimos con Perrenoud (2008) cuando plantea que todos los dispositivos de formación interactivos, y todas las formas de cooperación y de trabajo en equipo, pueden no sólo estimular una práctica reflexiva, sino también prepararla mediante la interiorización progresiva de comportamientos de clarificación, anticipación, justificación, inscriptos primero en un diálogo. 
Cabe aclarar que en este artículo en el que tratamos de desarrollar uno de los dispositivos de formación: la pareja pedagógica, trabajamos otros como la observación, el diario de clase, la biografía escolar, la microclase en forma separada para realizar un análisis más profundo de cada uno. Pero los dispositivos impactan en la formación docente cuando se articulan, cuando se entrelazan. Al respecto Anijovich (2009) sostiene:

Los dispositivos basados en interacciones privilegian el intercambio y la confrontación entre pares. Son propuestas que favorecen el ejercicio de la observación, el desarrollo de competencias comunicativas, la oferta y la recepción de retroalimentaciones, la integración de conocimientos de diferentes disciplinas y la articulación entre teoría y práctica. (p. 38)

A través de la inclusión de la pareja pedagógica como dispositivo dentro de los diferentes dispositivos para la formación docente intentamos pensar en una enseñanza orientada a "(...) considerar la participación activa y comprometida de los practicantes en su propio proceso de aprendizaje, alentándolos a expresar sus ideas, a desafiarse a sí mismos, a ir más allá y a disponerse a compartir y debatir sus propios saberes teóricos, sus producciones y sus experiencias" (Anijovich, et al., 2009, p. 166). La pareja pedagógica permite que el residente realice su residencia docente acompañado y no en soledad. Se concreta así desde la formación inicial, la socialización de las prácticas, instancia de valor inigualable para la comprensión y mejora de la misma.

\section{Referencias Bibliográficas}

- $\quad$ Aebli, H. (1995). Forma básica 3: contemplar y observar. En H. Aebli, 12 formas básicas de enseñar. Una didáctica basada en la psicología (pp.74-98) Madrid: Narcea.

- $\quad$ Alfonso, I. (2009). La investigación cualitativa como dispositivo de formación en las prácticas docentes. En L., Sanjurjo, A. (coord.), Caporossi, A., España, A. M., Hernández, I., Alfonso \& M. F Foresi, Los dispositivos para la formación en las prácticas profesionales (pp. 45-70) Rosario: Homo Sapiens.

- $\quad$ Alliaud, A. (2009). Los maestros a través del espejo. Una mirada desde la biografía escolar. En A., Alliaud, E., Antelo, Los gajes del oficio. Enseñanza, pedagogía y formación (51-63) Buenos Aires: Aique.

- $\quad$ Anijovich, R., Cappelletti, G., Mora, S. \& Sabelli, M. J. (2009). Experiencias de formación en la docencia. En R., Anijovich, G., Cappelletti, S., Mora \& M. J., Sabelli, Transitar la formación pedagógica. Dispositivos y estrategias (pp. 25-39) Buenos Aires: Paidós.

- Anijovich, R., Cappelletti, G., Mora, S. \& Sabelli, M. J. (2009). Autobiografía escolar: reconocer la propia historia de vida. En R., Anijovich, G., Cappelletti, S., Mora \& M. J., Sabelli, Transitar la formación pedagógica. Dispositivos y estrategias (pp. 83-99) Buenos Aires: Paidós.

- $\quad$ Anijovich, R., Cappelletti, G., Mora, S. \& Sabelli, M. J. (2009). Diarios de formación: el diálogo entre la subjetividad y la práctica. En R., Anijovich, G., Cappelletti, S., Mora \& M. J., Sabelli, Transitar la formación pedagógica. Dispositivos y estrategias (pp. 101-117) Buenos Aires: Paidós. 
- $\quad$ Anijovich, R., Cappelletti, G., Mora, S. \& Sabelli, M. J. (2009). La observación: educar la mirada para significar la complejidad. En R., Anijovich, G., Cappelletti, S., Mora \& M. J., Sabelli, Transitar la formación pedagógica. Dispositivos y estrategias (pp. 59-82) Buenos Aires: Paidós.

- $\quad$ Anijovich, R., Cappelletti, G., Mora, S. \& Sabelli, M. J. (2009). Microclases: prácticas simuladas de enseñanza. En R., Anijovich, G., Cappelletti, S., Mora \& M. J., Sabelli, Transitar la formación pedagógica. Dispositivos y estrategias (pp. 119-133) Buenos Aires: Paidós.

- $\quad$ Anijovich, R., Cappelletti, G., Mora, S. \& Sabelli, M. J. (2009). Reflexiones finales: Los dispositivos en acción. En R., Anijovich, G., Cappelletti, S., Mora \& M. J., Sabelli, Transitar la formación pedagógica. Dispositivos y estrategias (pp. 165-167) Buenos Aires: Paidós.

- $\quad$ Caporossi, A. (2009). La narrativa como dispositivo para la construcción del conocimiento profesional de las prácticas docentes. En L., Sanjurjo, A. (coord.), Caporossi, A., España, A. M., Hernández, I., Alfonso \& M. F Foresi, Los dispositivos para la formación en las prácticas profesionales (pp. 107-149) Rosario: Homo Sapiens.

- Devalle de Rendo, A. (2010). La residencia en la formación docente EGB 1 y 2. En A. Devalle de Rendo, La formación docente según las representaciones de los futuros maestros (pp. 19-34) Buenos Aires: Lugar.

- Hernández, A. M. (2009). El taller como dispositivo de formación y de socialización de las prácticas. En L., Sanjurjo, A. (coord.), Caporossi, A., España, A. M., Hernández, I., Alfonso \& M. F Foresi, Los dispositivos para la formación en las prácticas profesionales (pp. 71-106) Rosario: Homo Sapiens.

- $\quad$ Perrenoud, P. (2006). El análisis colectivo de la práctica como iniciación a la práctica reflexiva. En P. Perrenoud, Desarrollar la práctica reflexiva en el oficio de enseñar (pp. 115-136) España: Grao.

- Perrenoud, P. (2006). Introducción: La práctica reflexiva, clave de la profesionalización del oficio. En P. Perrenoud, Desarrollar la práctica reflexiva en el oficio de enseñar (pp. 9-27) España: Grao.

- $\quad$ Perrenoud, P. (2006). Saber reflexionar sobre la propia práctica: ¿es éste el objetivo fundamental de la formación de los enseñantes? En P. Perrenoud, Desarrollar la práctica reflexiva en el oficio de enseñar (pp. 45-67) España: Grao.

- $\quad$ Perrenoud, P. (2008). El trabajo sobre el habitus en la formación de maestros. Análisis de las prácticas y toma de conciencia. En L., Paquay, M., Altet, É., Charlier \& P. Perrenoud (coords.), La formación profesional del maestro. Estrategias y competencias (pp. 265-308) México: Fondo de Cultura Económica.

- Sanjurjo, L. (2005). Las teorías que fundamentan las prácticas reflexivas. En L. Sanjurjo, La formación práctica de los docentes. Reflexión y acción en el aula (pp. 19-54) Rosario: Homo Sapiens.

- Sanjurjo, L. (2009). Razones que fundamentan nuestra mirada acerca de la formación en las prácticas. En L., Sanjurjo, A. (coord.), Caporossi, A., España, A. M., Hernández, I., Alfonso \& M. F Foresi, Los dispositivos para la formación en las prácticas profesionales (pp. 15-43) Rosario: Homo Sapiens.

- $\quad$ Sanjurjo, L., Kahan, G., Hernández, A. M., Alfonso, I. \& Hernández, C. (2009) Proyecto de Trabajo. Universidad Nacional de Rosario, Facultad de Humanidades y Artes, Escuela de Ciencias de la Educación, Cátedra de Residencia Docente.

- Santomé, J. (1994). Prólogo: La práctica reflexiva y la comprensión de lo que acontece en las aulas. En Ph. W. Jackson, La vida en las aulas (pp.11-26) Madrid: Morata.

- Santos Guerra, M. A. (1998). Métodos de exploración. En M. A., Santos Guerra, Hacer visible lo cotidiano. Teoría y práctica de la evaluación cualitativa de los centros escolares (pp. 75-113) Madrid: Akal. 